\title{
Salvage prostate re-irradiation using high-dose-rate brachytherapy or focal stereotactic body radiotherapy for local recurrence after definitive radiation therapy
}

Aurélie Mbeutcha ${ }^{1,2}$, Laurent Chauveinc ${ }^{3}$, Pierre-Yves Bondiau', Marie-Eve Chand ${ }^{1}$, Matthieu Durand², Daniel Chevallier², Jean Amiel², Daniel Lam Cham Kee ${ }^{1}$ and Jean-Michel Hannoun-Lévi ${ }^{1 *}$

\begin{abstract}
Background: Optimal management of locally recurrent prostate cancer after definitive radiation therapy is still challenging. With the development of highly accurate radiotherapy devices, prostate salvage re-irradiation might generate lower toxicity rates than classical salvage therapies. We retrospectively evaluated the toxicity and the feasibility of a prostate re-irradiation after definitive radiation therapy failure. Two modalities were investigated: high-dose-rate brachytherapy (HDRB) on whole prostate gland and focal stereotactic radiotherapy (SBRT) using CyberKnife $\oplus^{\oplus i n a c . ~}$
\end{abstract}

Methods: Between 2011 and 2015, 28 patients with imaged and/or biopsy-proven intra-prostatic recurrence of cancer after definitive radiation therapy underwent a salvage re-irradiation using HDRB $(n=10)$ or focal SBRT $(n=18)$. The schedule of re-irradiation was $35 \mathrm{~Gy}$ in 5 fractions.

Biological response (defined as post-salvage radiation PSA variation) and biochemical no-evidence of disease (bNED) were evaluated in the whole cohort. For patients who had a positive biological response after salvage radiation, biochemical recurrence $(B C R)$ and survival after salvage radiotherapy were evaluated. Post-salvage toxicities were assessed according to the Common Terminology Criteria for Adverse Events (CTCAE) v4.03 and were compared to baseline status.

Results: Within a median follow-up of 22.5 months (IQR $=8-42), 9(90 \%)$ patients experienced a positive biological response after salvage HDRB and 5 (50\%) remained bNED at the end of the follow-up. Among patients who initially responded to salvage HDRB, the BCR rate was $44.4 \%$ after a median interval of 19.5 months $(\mathrm{IQR}=11.5-26)$. Only one patient experienced a transient grade 3 urinary complication.

In the SBRT group, the median follow-up was 14.5 months (IQR $=7-23)$ and $10(55.6 \%)$ out of the 18 patients remained bNED. Among the 15 patients who initially responded to salvage SBRT, 5 (33.3\%) experienced a BCR. One patient experienced a transient grade 4 urinary complication.

At the end of the follow-up, all evaluated patients had a urinary status grade variation $\leq+1$ grade. No grade $3-4$ digestive toxicity was observed.

(Continued on next page)

\footnotetext{
* Correspondence: jean-michel.hannoun-levi@nice.unicancer.fr

${ }^{1}$ Department of Radiation Oncology, Antoine-Lacassagne Cancer Canter, University of Nice Sophia-Antipolis, 33, avenue Valombrose, 06189, Nice Cedex 2, Nice, France

Full list of author information is available at the end of the article
} 
(Continued from previous page)

Conclusions: Salvage prostate re-irradiation for locally recurrent cancer is feasible and generate low toxicities rates when using with HDRB or focal SBRT. However, further investigations are necessary to confirm these findings and to determine predictive features for patients who might benefit from such an approach.

Keywords: Prostate cancer, Local recurrence, Salvage therapy, High-dose-rate brachytherapy, Stereotactic body radiation therapy, Cyberknife

\section{Introduction}

Although external beam radiation therapy (EBRT) and brachytherapy are very efficient treatment modalities for localized prostate cancer, more than the half of the patients would experience a biochemical recurrence (BCR) within 10 years $[1,2]$. Among these patients, about $20 \%$ would present a local recurrence for which most of the physicians would choose androgen-deprivation therapy (ADT) as the cornerstone of the therapy management [3]. But ADT remains a palliative treatment which significantly impacts quality of life [4]. Furthermore, tumor sensitivity to ADT is transient and cancer would eventually become castration-resistant. Therefore, preventing or delaying the introduction of ADT after PSA failure after initial treatment appears as a major challenge.

Local salvage procedures such as radical prostatectomy, high-intensity ultrasounds ablation (HIFU), cryosurgery or prostate re-irradiation [5-8] are therapy alternatives that can be offered to highly selected patients. Prostate reirradiation was mainly done with low-dose-rate brachytherapy using seeds, with mixed results on efficacy and toxicity $[5,9,10]$. However, recent improvement in radiotherapy devices such as high-dose rate brachytherapy (HDRB) and stereotactic body radiotherapy (SBRT) allow to deliver higher ablative dose in a smaller target volume with better sparing of surrounding critical organs at risk $[11,12]$. In spite of their encouraging results, very few patients benefit from these treatment options [3].

Actually, available data on post-radiation salvage HDRB and SBRT are very sparse and heterogeneous. Reported 2year BCR-free survival are around $50 \%$ for both radiation modalities [13-20]. The aim of this study was to evaluate the clinical outcomes (efficacy and toxicity) of salvage HDRB or SBRT (CyberKnife ${ }^{\bullet}$ Accuracy, Sunnyvale, CA, USA) in patients with post-radiation local recurrence of prostate cancer.

\section{Material and methods \\ Patients' selection}

We retrospectively reviewed medical records of patients treated with salvage HDRB or CyberKnife ${ }^{\circledR}$ for a postradiation prostate cancer local recurrence between 2011 and 2015 at Antoine-Lacassagne Cancer Center, Nice, France and at Clinique Hartmann, Levallois-Perret, France. All cases included in this study were discussed and approved by a multidisciplinary team. All patients provided a written informed consent for this institutions' review boards-approved study. A total of 43 patients were reviewed. Patients with proven lymph node and/or distant metastasis at the time of salvage radiation $(n=9)$, those who underwent another local treatment between primary and salvage radiation $(n=3)$, and patients with a follow-up shorter than 5 months $(n=3)$ were excluded from the study, leaving 28 patients to be included for final analysis (Fig. 1).

\section{Follow-up after primary radiation}

All patients experienced a BCR according to Phoenix criteria (PSA nadir $+2 \mathrm{ng} / \mathrm{mL}$ ) or 1997 ASTRO criteria (three consecutive rises of PSA level above the nadir). Imaging investigations following BCR included pelvic and prostatic MRI (T2-weighted, diffusion-weighted and dynamic contrast-enhanced), [11C]-choline positron emission computed tomography (PET-CT) and thorax, abdominal and pelvic CT-scan. The indications of prostate biopsy or ADT were retained after multidisciplinary discussion of each case. Salvage re-irradiation was considered only in patients with a relapse delay $>24$ months after initial radiation and a life-expectancy $>10$ years. Prostate biopsies were required before considering local salvage therapy. However, in case of histological impossibility to determine a precise Gleason score but with pathological features of malignancy together with a body of evidence in favor of a local recurrence (PSA kinetic, prostate MRI and [11C]-PET-CT findings) patients remained eligible for salvage radiation. Post-radiation complications were recorded using the Common Terminology Criteria for Adverse Events (CTCAE v4.03) [21].

\section{Salvage radiation modalities \\ Stereotactic body radiation therapy protocol}

Salvage SBRT was delivered with a CyberKnife ${ }^{\oplus}$ accelerator (Accuray, Sunnyvale, CA, USA). For tracking modality, at least three gold fiducials were placed in the prostate via trans-rectal ultrasonography (TRUS). Then, patients underwent a non-contrast-enhanced multi-slice CT scan (GE LightSpeed Scanner, GE Healthcare Diagnostic Imaging, Slough, UK) with $1.25 \mathrm{~mm}$ slice thickness. Patient immobilization during CT acquisition and treatment was obtained by a custom-build holding device. 


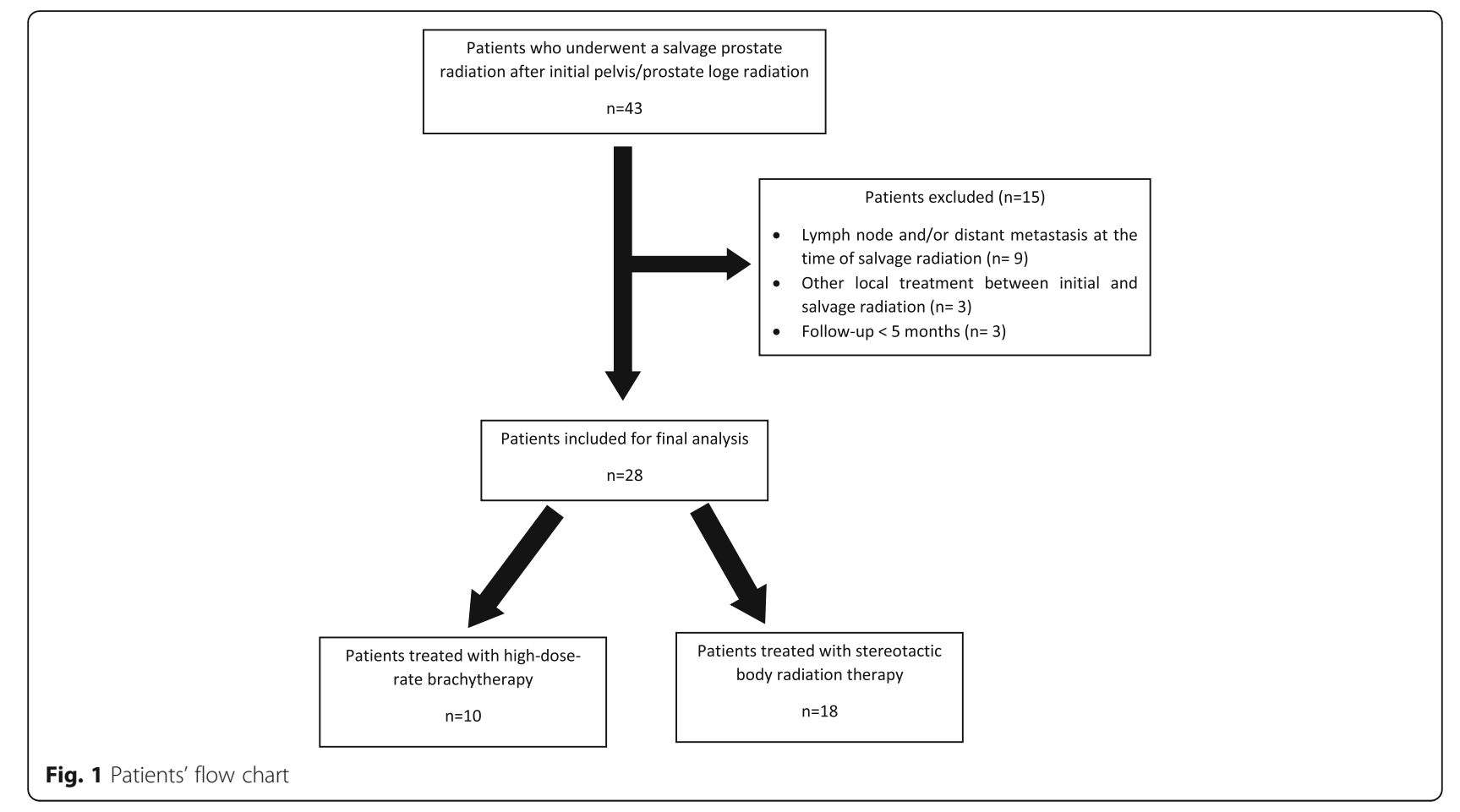

Prostate index tumor was defined by MRI and/or [11C]choline PET and was considered as the gross tumor volume (GTV). The GTV was delineated by using image fusion of MRI and/or [11C]-choline PET with planning CT. Assuming that the clinical target volume (CTV) would be the GTV plus a 1-mm margin, the planning target volume (PTV) was delineated as the CTV plus a $1-\mathrm{mm}$ margin leading to consider a focal SBRT. On each slice, contouring of organs at risk (rectum, bladder and femoral heads) were also performed. All plans were optimized using Multiplan ${ }^{\circ}$ treatment planning system (Accuray, Sunnyvale, CA, USA). The PTV received 35 Gy in 5 fractions of 7 Gy each in 5 consecutive days within the $80 \%$ coverage dose. The dose constraints for organs at risk were applied according to the American Association of Physicists in Medicine recommendations [22].

\section{High dose rate brachytherapy protocol}

The method of HDRB has been previously described [23]. Briefly, under general anesthesia and after urinary catheterization, 17 HDRB trans-perineal vectors (Sharp Needles $^{\mathrm{Tw}}$, Elekta AB, Stockholm, Sweden) were placed under TRUS guidance. The whole prostate gland and, if needed, the proximal part of the seminal vesicles were implanted using a dedicated perineal template. Due to vectors migration occurring after the implant [24], a new CT-scan was performed before each fraction allowing a specific re-planification and re-optimization of treatment plans. The CTV was defined as the outer contour of the prostate and included proximal part of the seminal vesicles if necessary. A total dose of 35 Gy was delivered in 5 fractions over 5 consecutive days. Urethra and rectum were delineated as organs at risk. According to recommendations of GEC/ESTRO [25], the following dose constrains for target volume were applied: the percentage of CTV receiving $100 \%$ of the prescribed isodose (V100) was $\geq 95 \%$; V150 was limited to $<30 \%$, and V200 was limited to $<12 \%$. The dose non-homogeneity ratio (DNR) had to be $<0.30$. For the urethra, V115 was kept to $<1 \%$ and for the rectum, V80 was kept to $<1 \%$. For both SBRT and HDRB, the doses received by organs at risk at initial treatment were not taken into account.

\section{Androgen deprivation therapy (ADT)}

At the time of recurrence, ADT was dispensed to 2 (20.0\%) patients that were further treated with salvage HDRB and one (10.0\%) of them did not discontinue his treatment before salvage HDRB. In the SBRT group, 10 (55.6\%) patients were receiving ADT before salvage treatment and for 3 (16.7\%) of them ADT was not withdrawn after salvage SBRT.

\section{Follow-up after salvage radiation and endpoints' definitions}

After salvage re-irradiation, PSA level dosage and clinical assessment of toxicities according to the CTCAE v4.03 were recorded at 2 months after treatment, then every 3 months for a year, then every 6 months. For the purpose of this study, biochemical response was defined according to PSA level variation $(\triangle \mathrm{PSA})$ between pre-salvage and 
post-salvage re-irradiation: biochemical positive response was defined as a diminution of $\triangle \mathrm{PSA}$ of more than $20 \%$, biochemical failure corresponded to $\triangle \mathrm{PSA}$ progressing of more than $20 \%$ and biochemical stabilization was defined between these two ranges.

In case of biochemical failure, a new [11C]-cholinePET-CT was performed to define local, regional or distant failure. A systemic treatment (ADT or chemotherapy) was introduced according to European Association of Urology guidelines [7]. For patients who experienced biochemical positive response or stabilization, post-salvage BCR was defined according to Phoenix criteria taking post-salvage PSA nadir level as a reference.

The primary endpoint was progression-free survival defined as biochemical no evidence of disease (bNED). For the group of patients who experienced a positive biochemical response or stabilization after salvage reirradiation, other analyzed survival covariates were BCRfree survival, local recurrence-free survival, lymph-node recurrence-free survival, metastatic-free survival and systemic therapy-free survival. Because some patients experienced late effects of the primary irradiation at the time of BCR and in order to assess the specific effect of prostate re-irradiation on organs at risk (urethra and rectum), acute ( $<3$ months) and late ( $>3$ months) toxicities were expressed as grade variations compared to baseline status using CTCAE v4.03 [21].

\section{Statistical analyses}

Continuous variables were expressed with median and interquartile range and were compared using the Kruskal-Wallis test. Categorical variables were compared with the $\chi 2$ test. Survival probabilities were estimated by the Kaplan-Meir method. Cases were censored at the last follow-up. A $p$-value $<0.05$ was considered significant. Statistical analyses were carried out using Stata v11 (College Station, TX, United States).

\section{Results}

\section{Patients' characteristics}

Finally, within a median follow-up of 15.5 months (IQR = 7-28), 28 patients were included in this study. Among them, 10 patients underwent HDRB while 18 patients underwent SBRT (Fig. 1). Table 1 summarizes patients' characteristics at primary treatment and at recurrence.

Patients treated with salvage SBRT had mainly received low-dose-rate brachytherapy (83.3\%) for low-risk group of D'Amico initial prostate cancer (55.6\%), whereas the HDRB group was mainly constituted of patients with initial high-risk disease (70.0\%) treated with EBRT (90.0\%) ( $p=0.006$ and $p<0.001$, respectively). The median time to BCR was 69 months $(\mathrm{IQR}=55-85)$ and 49 months (IQR $=37-70)$ for HDRB and SBRT groups, respectively $(p=0.11)$. There was no significant difference of delay between primary and salvage radiation in both groups (86.5 months (IQR = 66-108) vs. 77 months $(\mathrm{IQR}=64-92)$ for HDRB and SBRT respectively, $p=0.26$ ).

\section{Oncologic outcomes after salvage high-dose-rate brachytherapy}

Ten patients underwent salvage HDRB within a median follow-up of 22.5 months (IQR $=8-42)$ and 5 (50\%) remained bNED at the end of the follow-up. Dosimetric features of HDRB are resumed in Table 2a. The median PSA level at the time of salvage HDRB was $4.42 \mathrm{ng} / \mathrm{mL}$ $(\mathrm{IQR}=2.01-6.7)$. Nine $(90 \%)$ patients had a biochemical positive response with a median $\triangle \mathrm{PSA}$ of $-2.81 \mathrm{ng} / \mathrm{mL}$ (IQR = -5.6;-1.16) and 1 (10\%) patient experienced a biochemical progression despite salvage HDRB. For patients who responded to salvage $\operatorname{HDRB}(n=9,90 \%)$, median PSA nadir was $0.66 \mathrm{ng} / \mathrm{mL}(\mathrm{IQR}=0.23-1.13)$ and was reached within 6 months $(\mathrm{IQR}=5-8)$ after salvage radiation. Among patients who initially experienced biochemical positive response to $\operatorname{HDRB}(n=9,90 \%), 4$ (44.4\%) of them experienced BCR within 19.5 months $(\mathrm{IQR}=11.5-26)$ after salvage therapy (Fig. 2). Three (33.3\%) patients experienced image-proven loco-regional disease recurrence within 27 months (IQR = 14-36) and 2 patients (22.2\%) experienced metastatic disease progression within a median time interval of 25 months $(\mathrm{IQR}=14-36)$. When including patients who did not interrupted their ADT, median time before the introduction of systemic treatment was 14 months (IQR $=6-27)$. With a follow-up of 21 months, the patient who experienced immediate HDRB biochemical failure did not developed extra-prostatic disease progression after introduction of ADT.

\section{Oncologic outcomes after salvage stereotactic body radiation therapy}

Within a median follow-up of 14.5 months (IQR $=7-23$ ), $10(55.6 \%)$ out of the 18 patients who underwent salvage SBRT remained bNED. SBRT dosimetric characteristics are resumed in Table $2 \mathrm{~b}$. The median PSA level at the time of salvage SBRT was $4.5 \mathrm{ng} / \mathrm{ml}(\mathrm{IQR}=3.0-5.3)$. Thirteen patients $(72.2 \%)$ presented a regression of PSA level after salvage SBRT with a median $\triangle \mathrm{PSA}$ of $-3.2 \mathrm{ng} / \mathrm{mL}(\mathrm{IQR}=-5.34 ;-1.8), 2$ patients $(11.1 \%)$ had a PSA level stabilization and $3(16.7 \%)$ patients experienced post-SBRT biochemical failure. When considering patients who had a positive response or stabilization $(n=15)$, nadir PSA level was $1.00 \mathrm{ng} / \mathrm{mL}(\mathrm{IQR}=0.42-2.4)$ and was reached within 7.5 months $(\mathrm{IQR}=4.5-10)$ after salvage SBRT. Five patients (33.3\%) experienced BCR within a median follow-up of 7 months (IQR $=4-7$ ) (Fig. 2). Among them, 3 patients (20.0\%) experienced loco-regional disease recurrence within 5 months (IQR = 4.9-9.5) and 1 (6.7\%) had a metastatic progression of his 
Table 1 Patients' characteristics

\begin{tabular}{|c|c|c|c|c|c|c|}
\hline \multirow[t]{2}{*}{ Characteristics } & \multicolumn{2}{|c|}{$\begin{array}{l}\text { High-dose-rate brachytherapy } \\
(n=10)\end{array}$} & \multicolumn{2}{|c|}{$\begin{array}{l}\text { Stereotactic body radiation therapy } \\
(n=18)\end{array}$} & \multirow{2}{*}{$\begin{array}{l}p \text { value } \\
\text { Primary }\end{array}$} & \multirow{2}{*}{$\begin{array}{l}p \text { value } \\
\text { Recurrence }\end{array}$} \\
\hline & Primary treatment (\%) & Recurrence (\%) & Primary treatment (\%) & Recurrence (\%) & & \\
\hline Age (years, median, IQR) & $63(58-68)$ & $69(65-77)$ & $62(58-66)$ & $69(64-75)$ & 0.87 & 0.85 \\
\hline $\begin{array}{l}\text { PSA at the time of treatment } \\
(\mathrm{ng} / \mathrm{mL} \text {, median, lQR) }\end{array}$ & $26(8.6-47)$ & $4.37(2.01-4.76)$ & $6.6(5.7-9.2)$ & $4.5(3.0-6.3)$ & 0.0046 & 0.43 \\
\hline Initial T stage & & - & & - & 0.005 & \\
\hline T1c & $2(20.0)$ & & $12(66.7)$ & & & \\
\hline $\mathrm{T} 2 \mathrm{a}$ & $2(20.0)$ & & $2(11.1)$ & & & \\
\hline T3 & $6(60.0)$ & & $2(11.1)$ & & & \\
\hline Not evaluated & - & & $2(11.1)$ & & & \\
\hline Initial Gleason sum & & & & & 0.04 & 0.71 \\
\hline Gleason 6 or less & $2(20.0)$ & $1(10.0)$ & $13(72.2)$ & $2(11.1)$ & & \\
\hline Gleason 7 & $4(40.0)$ & $2(20.0)$ & $4(22.2)$ & $2(11.1)$ & & \\
\hline Gleason 8 & $4(40.0)$ & $1(10.0)$ & $1(5.6)$ & $1(5.6)$ & & \\
\hline Gleason 9 & - & $2(20.0)$ & - & $2(11.1)$ & & \\
\hline Not evaluated & - & $4(40.0)$ & - & $11(61.1)$ & & \\
\hline D'Amico risk group & & & & & 0.006 & \\
\hline Low & $1(10.0)$ & - & $10(55.6)$ & - & & \\
\hline Intermediate & $2(20.0)$ & & $5(27.8)$ & & & \\
\hline High & $7(70.0)$ & & $2(11.1)$ & & & \\
\hline Not evaluated & - & & $1(5.6)$ & & & \\
\hline Tumor localization & & & & & 0.21 & 0.019 \\
\hline Apex & - & $1(10.0)$ & - & & & \\
\hline Median & - & - & $2(11.1)$ & $2(11.1)$ & & \\
\hline Basis & - & $3(30.0)$ & $1(5.6)$ & $1(5.6)$ & & \\
\hline Seminal vesical & $1(10.0)$ & - & - & $7(38.9)$ & & \\
\hline More than 1 location & $1(10.0)$ & $6(60.0)$ & $3(16.7)$ & $3(16.7)$ & & \\
\hline Not evaluated & $8(80.0)$ & $1(10.0)$ & $12(66.7)$ & $4(22.2)$ & & \\
\hline Initial ADT duration & & - & & - & 0.0031 & \\
\hline Short (6 months) & $2(20.0)$ & & $7(38.9)$ & & & \\
\hline Long (24-36 months) & $5(50.0)$ & & $1(5.6)$ & & & \\
\hline No ADT & $3(30.0)$ & & $10(55.6)$ & & & \\
\hline ADT at recurrence & - & & - & & & 0.05 \\
\hline Yes & & $2(20.0)$ & & $10(55.6)$ & & \\
\hline No & & $8(80.0)$ & & $8(44.4)$ & & \\
\hline ADT duration (months, median, IQR) & - & $63.5(48-79)$ & - & $15(6-21)$ & & 0.028 \\
\hline Initial radiation modality & & - & & - & $<0.001$ & \\
\hline LDR brachytherapy & $1(10.0)$ & & $15(83.3)$ & & & \\
\hline EBRT w/o pelvic radiation & $5(50.0)$ & & 3 (16.7)- & & & \\
\hline EBRT with pelvic radiation & $4(40.0)$ & & & & & \\
\hline Prostate volume (cc, median, IQR) & $33.5(32-35)$ & $35(20-50)$ & $35(25-44.5)$ & $26(22.5-28.5)$ & 0.78 & 0.59 \\
\hline
\end{tabular}


Table 1 Patients' characteristics (Continued)

\begin{tabular}{|c|c|c|c|c|c|c|}
\hline PSA nadir (ng/mL, median, IQR) & $0.065(0.01-0.2)$ & $0.66(0.23-1.13)$ & $0.58(0.34-1.05)$ & $0.89(0.29-1.4)$ & 0.0034 & 0.97 \\
\hline Time to PSA nadir (months, median, IQR) & $21(16-30)$ & $6(5-8)$ & $28(11-35)$ & $7.5(4.5-10)$ & 0.79 & 0.89 \\
\hline $\begin{array}{l}\text { Time to biological recurrence } \\
\text { (months, median, IQR) }\end{array}$ & $69(55-85)$ & $13(10-26)$ & $49(37-70)$ & $5.5(4-6)$ & 0.11 & 0.0347 \\
\hline $\begin{array}{l}\text { Time to salvage treatment } \\
\text { (months, median, IQR) }\end{array}$ & $86.5(66-108)$ & - & $77(64-92)$ & - & 0.26 & \\
\hline
\end{tabular}

Note: PSA prostate specific antigen, $A D T$ androgen deprivation therapy, IQR interquartile range, $L D R$ low-dose-rate, EBRT external beam radiotherapy

disease. All the 3 patients who experienced a post-SBRT biochemical failure developed an extra-prostatic evolution despite the introduction of ADT.

\section{Toxicities after salvage radiation}

Toxicities following salvage radiation are summarized in Tables 3.

During the course of HDRB and within 3 months thereafter, most patients experienced grade 1-2 toxicities (Table 3a). When comparing to baseline, 2 patients (20\%) had no modification of their acute urinary status, 3 (30\%) experienced an increase of +1 grade and $4(40 \%)$ of +2 grades.

Table 2 Dosimetric features for high-dose-rate brachytherapy (a) and cyberknife (b)

\begin{tabular}{|c|c|c|c|}
\hline \multicolumn{4}{|l|}{ a } \\
\hline Parameter & \multicolumn{3}{|l|}{ Value } \\
\hline CTV & \multicolumn{3}{|l|}{$35(25-43)$} \\
\hline D90 & \multicolumn{3}{|c|}{$106(106-108)$} \\
\hline D100 & \multicolumn{3}{|l|}{$81(77-85)$} \\
\hline V100 & \multicolumn{3}{|l|}{97 (96-98) } \\
\hline V150 & \multicolumn{3}{|l|}{$24(22-32)$} \\
\hline V200 & \multicolumn{3}{|l|}{$8(7-10)$} \\
\hline D2 rectum & \multicolumn{3}{|l|}{$57(50-69)$} \\
\hline D2 urethra & \multicolumn{3}{|l|}{$81(76-85)$} \\
\hline $\mathrm{DHI}$ & \multicolumn{3}{|c|}{$0.27(0.22-0.33)$} \\
\hline \multicolumn{4}{|l|}{$b$} \\
\hline Region of interest & Mean (cGy) & Min (cGy) & Max (cGy) \\
\hline GTV & 4027 & 3462 & 4375 \\
\hline PTV & 3936 & 3134 & 4375 \\
\hline Bladder & 862 & 161 & 3680 \\
\hline Rectum & 727 & 102 & 3561 \\
\hline Right femoral head & 371 & 145 & 894 \\
\hline Left femoral head & 428 & 139 & 1075 \\
\hline
\end{tabular}

Note: CTV clinical target volume, D90 dose delivered to $90 \%$ of the CTV, D100 dose delivered to $100 \%$ of the CTV, V100 volume receiving $100 \%$ of the prescribed dose, $V 150$ volume receiving $150 \%$ of the prescribed dose, V200 volume receiving $200 \%$ of the prescribed dose, D2 rectum dose delivered to $2 \%$ of the rectum volume, $D 2$ urethra dose delivered to $2 \%$ of the urethra volume, $D H I$ dose non-homogeneity Index: V150/V100

Note: GTV gross target volume, PTV planning target volume
When considering late toxicities, 5 patients $(50 \%)$ had a urinary condition worsen of +1 grade and 1 patient $(10 \%)$ had $\mathrm{a}+2$ grade worse condition (Table $3 \mathrm{~b})$. One $(10 \%) \mathrm{pa}-$ tient experienced a grade 3 hematuria 12 months after salvage HDRB, treated by hyperbaric chamber. No grade 3-4 digestive toxicity occurred during the follow-up.

After salvage SBRT, no patient experienced grade 3-4 digestive toxicity (Table 3c). One patient (5.6\%) experienced a septic shock following a prostatitis complicated by a histology-proven prostate necrosis 12 months after salvage SBRT. This patient already had a history of prostatitis and underwent several transurethral resections of the prostate for benign prostatic obstruction. At last follow-up, 12 patients (66.7\%) had no modification of their urinary and digestive status compared to baseline (Table 3d).

\section{Discussion}

Despite its wide range of side effects and the absence of curative perspective, ADT remains the most chosen option in case of local recurrence of prostate cancer after definitive radiation therapy $[3,4]$. However, international guidelines also consider the possibility to offer a local salvage therapy to some patients who meet the criteria of a strictly intra-prostatic disease [7]. For these highly selected patients (PSA $<10 \mathrm{ng} / \mathrm{ml}$, Gleason score $\leq 7$, PSA doubling time $\geq 16$ months and life expectancy $>10$ years), a salvage treatment modality can be proposed in order to control the local disease, and delay, or better still, suppress the indication of ADT.

Due to the reluctance of physicians to intervene on potentially vulnerable tissues, local salvage options are hardly ever proposed to patients [3]. In this context, salvage prostate re-irradiation can only be considered and investigated if this option is associated with low toxicity rates.

Therefore, in this retrospective study, we evaluated the toxicity and the feasibility of a prostate re-irradiation after definitive radiation therapy failure. Two modalities were investigated: HDRB and SBRT using CyberKnife ${ }^{\circ}$.

Available data on salvage re-irradiation with HDRB are sparse and were mainly limited by their heterogeneity for both the type of patients included and the radiation protocols used [13-16, 26]. Furthermore, they were 


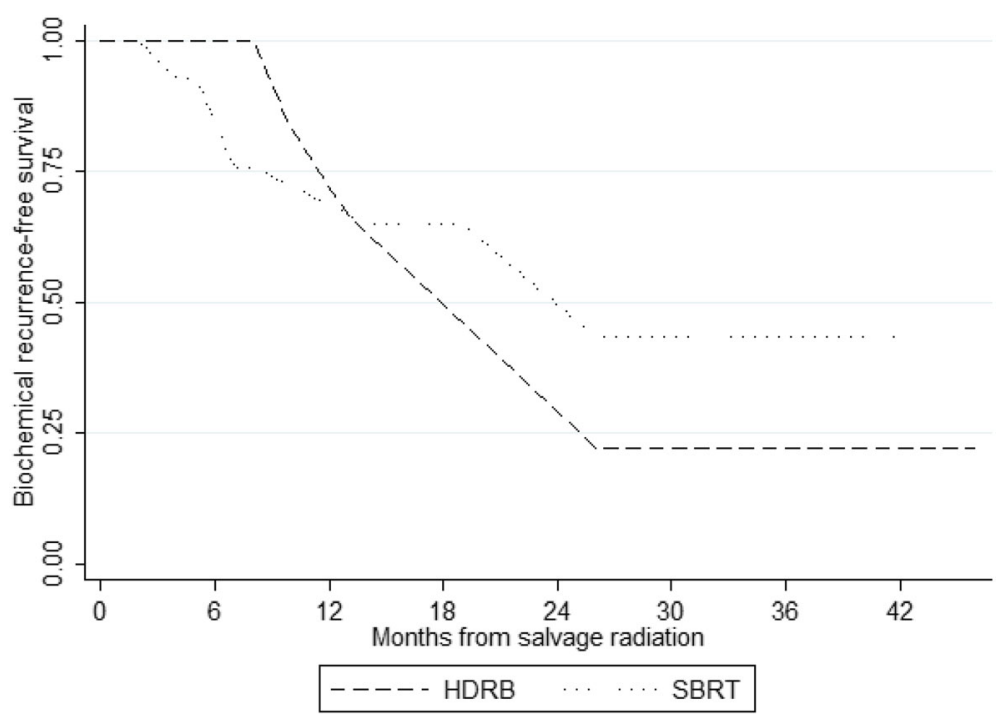

Fig. 2 Biochemical recurrence-free survival after salvage high-dose-rate brachytherapy $(n=10)$ and stereotactic body radiation therapy $(n=18)$

often conducted on small cohorts. However, reported 2year bNED or BCR-free survivals ranged from 50 to $89 \%$. To date, only one prospective study is available. In this phase II study, Yamada et al. treated 42 patients with a total dose of 32 Gy in 4 fractions [17]. Within a median follow-up of 36 months, $68.5 \%$ remained free of BCR.

After definitive radiation therapy for the primary prostate cancer, late grade $1-2$ and 3-4 urinary complications are about $17 \%$ and $3 \%$, respectively. For digestive toxicity, $15 \%$ of the patients would experience grade $1-2$ complications and $2 \%$ of them would experience grade 3-4 adverse events [27]. Logically, at the time of local salvage re-irradiation on potentially weakened tissues, higher morbidity rates are expected whatever the chosen option is. This is also true for salvage prostatectomy which is associated with the highest rates of complications, even if it remains the most efficient option for oncologic outcomes [28-30]. Whole prostate gland cryosurgery and low-dose-rate brachytherapy with seeds have similar survival rates with HDRB, but are associated with poorer functional outcomes $[28,30]$. Indeed, the most recently published protocols of HDRB were associated with a grade 3 urinary complication rate between 2 and $20 \%$, with almost none severe digestive complications $[13,15-17,26]$. With a bNED rate of $50 \%$ after 22.5 months of median follow-up and very low severe toxicity rates, our results on salvage HDRB are very encouraging and are comparable to published data. In this context, it is worth proposing a prostate re-irradiation to some highly selected patients.

To date, only few studies have been published on salvage re-irradiation using a CyberKnife ${ }^{\bullet}$ linac. Vavassori et al. published the first results on prostate re-irradiation using this system. In this preliminary retrospective case series including 6 patients treated with a total dose of 30 Gy in 5 fractions on the whole prostate gland, 2 patients remained bNED after 11.2 months of median follow-up [18]. Since then, Fuller et al. published a retrospective study including 29 patients treated with a total dose of 34 Gy in 5 fractions within a median follow-up of 24 months. They reached an actuarial 2-year BCR-free survival of $82 \%$ with only $7 \%$ of grade $3-4$ urinary toxicity and no severe digestive toxicity [20].

Our approach for salvage SBRT was slightly different as we used focal re-irradiation instead of whole gland treatment. We reached a bNED rate of $55.6 \%$ at 14.5 months of median follow-up and a BCR-free survival of $66.7 \%$ with reasonable induced toxicity. Janoray et al. recently published a report on focal salvage SBRT using CyberKnife ${ }^{\bullet}$ after EBRT failure [31]. In this retrospective study including 11 patients, they reached a biochemical response rate of $82 \%$ ( 9 out of 11 patients). At twelve months of follow-up, 6 patients were free of disease recurrence. Our results on survival outcomes are similar to those obtained by other focal salvage therapies such as cryosurgery, HIFU or low-dose-rate brachytherapy. Reported 1-year BCR-free survival ranged from 69 to $100 \%$ and dropped to $49-100 \%$ at 2 -year [32]. Severe reported urinary and digestive toxicities ranged from 0 to $33.3 \%$. In our study, most of the patients had transient low-grade complications and returned to baseline at the last follow-up.

Therefore, both salvage HDRB and focal SBRT appear as suitable options to delay the introduction of ADT in some highly selected patients, without exposing patients 
Table 3 Complication grades and grade variations following salvage high-dose-rate brachytherapy (a, b) and stereotactic body radiation therapy (c, d) using CTCAE v4.03

\begin{tabular}{|c|c|c|c|c|}
\hline \multicolumn{5}{|l|}{$\bar{a}$} \\
\hline HDRB & At baseline & Maximal acute toxicity & Maximal late toxicity & At last follow-up \\
\hline \multicolumn{5}{|c|}{ Urinary complication (n, \%) } \\
\hline No complication & $5(50.0)$ & - & $2(20.0)$ & $2(20.0)$ \\
\hline Grade 1 & $4(40.0)$ & $2(20.0)$ & - & $3(30.0)$ \\
\hline Grade 2 & $1(10.0)$ & $7(70.0)$ & $6(60.0)$ & $5(50.0)$ \\
\hline Grade 3 & - & - & $1(10.0)$ & - \\
\hline Grade 4 & - & - & - & - \\
\hline Not evaluated & & $1(10.0)$ & $1(10.0)$ & - \\
\hline \multicolumn{5}{|c|}{ Digestive complication (n, \%) } \\
\hline No complication & $7(70.0)$ & $8(80.0)$ & $7(70.0)$ & $10(100.0)$ \\
\hline Grade 1 & $3(30.0)$ & $1(10.0)$ & $2(20.0)$ & - \\
\hline Grade 2 & - & - & - & - \\
\hline Grade 3 & - & - & - & - \\
\hline Grade 4 & - & - & - & - \\
\hline Not evaluated & - & $1(10.0)$ & $1(10.0)$ & - \\
\hline \multicolumn{5}{|l|}{$b$} \\
\hline \multirow[t]{2}{*}{ HDRB } & At 3 months & & At last follow-up & \\
\hline & $\mathrm{n}$ & $\%$ & $n$ & $\%$ \\
\hline \multicolumn{5}{|l|}{ Urinary complication } \\
\hline No modification & 2 & 20.0 & 4 & 40.0 \\
\hline+1 grade & 3 & 30.0 & 5 & 50.0 \\
\hline+2 grades & 4 & 40.0 & 1 & 10.0 \\
\hline Not evaluated & 1 & 10.0 & - & - \\
\hline \multicolumn{5}{|c|}{ Digestive complication } \\
\hline No modification & 9 & $90.0 \%$ & 10 & 100 \\
\hline+1 grade & - & - & - & - \\
\hline+2 grades & - & - & - & - \\
\hline Not evaluated & 1 & $10.0 \%$ & - & - \\
\hline \multicolumn{5}{|l|}{ c } \\
\hline SBRT & At baseline & Maximal acute toxicity & Maximal late toxicity & At last follow-up \\
\hline \multicolumn{5}{|c|}{ Urinary complication (n, \%) } \\
\hline No complication & $8(44.4)$ & $2(11.1)$ & $6(33.3)$ & $4(22.2)$ \\
\hline Grade 1 & $5(27.8)$ & $5(27.8)$ & $4(22.2)$ & $6(33.3)$ \\
\hline Grade 2 & $3(16.7)$ & $2(11.1)$ & $1(5.6)$ & $3(16.7)$ \\
\hline Grade 3 & $1(5.6)$ & - & - & - \\
\hline Grade 4 & - & - & $1(5.6)$ & - \\
\hline Not evaluated & $1(5.6)$ & $9(50.0)$ & $6(33.3)$ & $5(27.8)$ \\
\hline \multicolumn{5}{|c|}{ Digestive complication ( $n, \%)$} \\
\hline No complication & $13(72.2)$ & $5(27.8)$ & $9(50.0)$ & $10(55.6)$ \\
\hline Grade 1 & $1(5.6)$ & $1(5.6)$ & - & $1(5.6)$ \\
\hline Grade 2 & $2(11.1)$ & $2(11.1)$ & $1(5.6)$ & $2(11.1)$ \\
\hline Grade 3 & - & - & - & - \\
\hline
\end{tabular}


Table 3 Complication grades and grade variations following salvage high-dose-rate brachytherapy (a, b) and stereotactic body radiation therapy (c, d) using CTCAE v4.03 (Continued)

\begin{tabular}{|c|c|c|c|c|}
\hline Grade 4 & - & - & - & - \\
\hline Not evaluated & $2(11.1)$ & $10(55.6)$ & $8(44.4)$ & $5(27.8)$ \\
\hline \multicolumn{5}{|l|}{$d$} \\
\hline \multirow[t]{2}{*}{ SBRT } & \multicolumn{2}{|l|}{ At 3 months } & \multicolumn{2}{|l|}{ At last follow-up } \\
\hline & $n$ & $\%$ & $n$ & $\%$ \\
\hline \multicolumn{5}{|l|}{ Urinary complication } \\
\hline No modification & 4 & 22.3 & 12 & 66.7 \\
\hline+1 grade & 4 & 22.2 & 1 & 5.6 \\
\hline+2 grades & 1 & 5.6 & - & - \\
\hline Not evaluated & 9 & 50.0 & 5 & 27.8 \\
\hline \multicolumn{5}{|c|}{ Digestive complication } \\
\hline No modification & 7 & 38.9 & 12 & 66.7 \\
\hline+1 grade & - & - & - & - \\
\hline+2 grades & 1 & 5.6 & - & - \\
\hline Not evaluated & 10 & 55.6 & 6 & 33.3 \\
\hline
\end{tabular}

to an unreasonable risk of complication. But the scope of our results is limited by the retrospective setting of our study, its small cohort and its short follow-up that make it not calibrated to establish cancer-specific and overall survival rates. Furthermore, even if few patients did not discontinue their ADT after salvage radiation, this fact constitutes a bias for the interpretation of survival outcomes. Indeed, the heterogeneous population including both hormone-refractory and hormone-naïe prostate cancers together with both low- and high-grade cancers probably impacted oncologic outcomes. Therefore, the determination of predictive factors for radiation sensitive cancers would help to refine the selection of patients who could benefit from a salvage prostate reirradiation. These questions have been discussed during the Delphi conference of consensus on salvage brachytherapy [33], but many crucial questions such as maximal PSA value at the time of salvage brachytherapy, prostate gland volume to treat (whole gland, half or focal) or radiation doses did not reach a consensus.

The choice to perform a focal radiation with CyberKnife ${ }^{\oplus}$ has been encouraged by oncologic results of focal cryosurgery and HIFU which were comparable to whole gland treatment with less toxicity [32]. But as all focal treatments, the main limitation remains in the definition of the target volume. Even if we used fusion of MRI and [11C]-choline-PET, we can't guaranty that the visualized lesions are the only cancerous spots in the prostate.

But this study has also strengths: our hypo-fractionation scheme for HDRB allowed, contrarily to most of the studies published on this topic, to treat patients within 5 consecutive days with only one implant. This report on salvage focal CyberKnife ${ }^{\bullet}$ shows promising results that would worth to be confirmed in a larger and prospective manner.

\section{Conclusion}

For highly selected patients, salvage prostate re-irradiation using HDRB or focal SBRT are suitable options to treat local recurrence of cancer after definitive radiation therapy. The low toxicity rates associated with both of these techniques are encouraging proposing these options to a well-defined group of patients, without exposing them to an unreasonable risk. However, further investigations are needed to confirm these findings and to define selection criteria for patients who could benefit from such approaches.

\section{Abbreviations}

ADT: Androgen-deprivation therapy; BCR: Biochemical recurrence; bNED: Biological non-evidence of disease; CT: Computerized tomography; CTCAE: Common Terminology Criteria for Adverse Events; CTV: Clinical target volume; EBRT: External beam therapy; GTV: Gross target volume; HDRB: High-dose rate brachytherapy; HIFU: High-intensity ultrasounds ablation; IQR: Interquartile range; MRI: Magnetic resonance imaging; PET: Positron emission computed tomography; PSA: Prostate specific antigen; PTV: Planning target volume; SBRT: Stereotactic body radiotherapy; TRUS: Trans-rectal ultrasonography

\section{Acknowledgements}

Not applicable.

Funding

Not applicable.

Availability of data and materials

The dataset analyzed during the current study is available from the corresponding author on reasonable request. 


\section{Competing interests}

The authors declare that they have no competing interest.

\section{Authors' contributions}

AM collected, managed and analyzed data, wrote and edited the manuscript; LC developed the project, collected and managed data and edited the manuscript; PYB developed the project, collected and managed data and edited the manuscript; MEC collected data and edited the manuscript; MD edited the manuscript; DC edited the manuscript; JA edited the manuscript; DLCK collected and managed data and participated to manuscript editing; JMHL developed the project, collected and managed data, participated to data analysis, wrote and edited the manuscript. All authors read and approved the final manuscript.

\section{Consent for publication}

Not applicable.

\section{Ethics approval and consent to participate}

This study has been approved by both institutions' review boards. All patients provided written informed consent.

\section{Author details}

'Department of Radiation Oncology, Antoine-Lacassagne Cancer Canter, University of Nice Sophia-Antipolis, 33, avenue Valombrose, 06189, Nice Cedex 2, Nice, France. ${ }^{2}$ Department of Urology, Hôpital Pasteur 2, Centre Hospitalier Universitaire de Nice, University of Nice Sophia-Antipolis, Nice, France. ${ }^{3}$ Department of Radiation Oncology, Clinique Hartmann, Levallois-Perret, France.

\section{Received: 15 September 2016 Accepted: 22 February 2017}

\section{Published online: 09 March 2017}

\section{References}

1. Neppl-Huber C, et al. Changes in incidence, survival and mortality of prostate cancer in Europe and the United States in the PSA era: additional diagnoses and avoided deaths. Ann Oncol. 2012:1325-1334. doi:10.1093/annonc/mdr414.

2. Agarwal PK, Sadetsky N, Konety BR, Resnick MI, Carroll PR. Treatment Failure After Primary and Salvage Therapy for Prostate Cancer: likehood, patterns of care and outcomes. Cancer. 2007;112.

3. Tran H, Kwok J, Pickles T, Tyldesley S, Black PC. Underutilization of local salvage therapy after radiation therapy for prostate cancer. Urol Oncol. 2014:32:701-6.

4. Ahmadi H, Daneshmand S. Androgen deprivation therapy : evidence-based management of side effects. BJU Int. 2013:543-548. doi:10.1111/j.1464-410X 2012.11774.x.

5. Ramey SJ, Marshall DT. Re-irradiation for salvage of prostate cancer failures after primary radiotherapy. World J Urol. 2013;31:1339-45.

6. Boukaram C, Hannoun-Levi J. Management of prostate cancer recurrence after definitive radiation therapy. Cancer Treat Rev. 2010;36:91-100.

7. Heidenreich A, et al. EAU Guidelines on Prostate Cancer. Part II : Treatment of Advanced, Relapsing, and Castration-Resistant Prostate Cancer. Eur Urol. 2013;65:467-79.

8. Nguyen PL, D'Amico AV, Lee AK, Suh WW. Patient Selection, Cancer Control, and Complications After Salvage Local Therapy for Postradiation Prostatespecific Antigen Failure/A Sytematic Review of the Literature. Cancer. 2007; 110:1417-28.

9. Henríquez I, et al. Salvage brachytherapy in prostate local recurrence after radiation therapy: predicting factors for control and toxicity. Radiat Oncol. 2014;9:102.

10. Vargas $C$, et al. Salvage brachytherapy for recurrent prostate cancer. Brachytherapy. 2014;13:53-8.

11. Fukuda $\mathrm{S}$, et al. Dosimetry analyses comparing high-dose-rate brachytherapy, administered as monotherapy for localized prostate cancer with stereotactic body radiation therapy simulated using Cyberknife. J Radiat Res. 2014;55:1114-21.

12. Sudahar H, Kurup PGG, Murali V, Mahadev P, Velmurugan J. Medica Dosimetry Analysis of high - dose rate brachytherapy dose distribution resemblance in CyberKnife hypofractionated treatment plans of localized prostate cancer. Med Dosim. 2013;38:385-9.
13. Chen $C P$, et al. Salvage HDR brachytherapy for recurrent prostate cance after previous definitive radiation therapy: 5-year outcomes. Int J Radiat Oncol Biol Phys. 2013;86:324-9.

14. Jo Y, et al. Salvage high-dose-rate brachytherapy for local prostate cancer recurrence after radiotherapy - preliminary results. BJU Int. 2011;109:835-9.

15. Lee $B$, et al. Feasibility of high-dose-rate brachytherapy salvage for local prostate cancer recurrence after radiotherapy: the university of CaliforniaSan Francisco experience. Int J Radiat Oncol Biol Phys. 2007;67:1106-12.

16. Tharp $M$, et al. Prostate high-dose-rate brachytherapy as salvage treatment of local failure after previous external or permanent seed irradiation for prostate cancer. Brachytherapy. 2008;7:231-6.

17. Yamada Y, et al. A Phase II study of salvage high-dose-rate brachytherapy for the treatment of locally recurrent prostate cancer after definitive external beam radiotherapy. Brachytherapy. 2014;13:111-6.

18. Vavassori A, et al. Image-guided robotic radiosurgery as salvage therapy for locally recurrent prostate cancer after external beam irradiation : retrospective feasibility study on six cases. Tumori. 2010;96:71-5.

19. Zerini $D$, et al. Salvage image-guided intensity modulated or stereotactic body reirradiation of local recurrence of prostate cancer. Br J Radiol. 2015; 88:20150197.

20. Fuller DB, Wurzer J, Shirazi R, Bridge SS, Law J, Mardirossian G. High-doserate stereotactic body radiation therapy for postradiation therapy locally recurrent prostatic carcinoma: Preliminary prostate-specific antigen response, disease-free survival, and toxicity assessment. Pract Radiat Oncol. 2015;5(6):e615-23.

21. National Cancer Institute, Common Terminology Criteria for Adverse Events v4.03, NCl, NIH. DHHS. 2009. http://www.hrc.govt.nz/sites/default/files/ CTCAE\%20manual\%20-\%20DMCC.pdf. Accessed 4 Mar 17.

22. Benedict $\mathrm{SH}$, et al. Stereotactic body radiation therapy: the report of AAPM Task Group 101. Med Phys. 2010;37:4078-101.

23. Claren A, Gautier M, Feuillade J, Falk AT, Hannoun-Levi JM. Second salvage treatment for local recurrence of prostate cancer using high-dose-rate brachytherapy: A case report. J Contemp Brachytherapy. 2015;7:244-7.

24. Simnor $\mathrm{T}$, et al. Justification for inter-fraction correction of catheter movement in fractionated high dose-rate brachytherapy treatment of prostate cancer. Radiother Oncol. 2009;93:253-8.

25. Hoskin PJ, et al. GEC/ESTRO recommendations on high dose rate afterloading brachytherapy for localised prostate cancer: an update. Radiother Oncol. 2013;107:325-32.

26. Wojcieszek P, Szlag M, Głowacki G, Cholewka A. Salvage high-dose-rate brachytherapy for locally recurrent prostate cancer after primary radiotherapy failure. Radiother Oncol. 2016;119:405-10.

27. Ohri N, Dicker AP, Showalter TN. Late toxicity rates following definitive radiotherapy for prostate cancer. Can J Urol. 2012;19:6373-80.

28. Parekh A, Graham PL, Nguyen PL. Cancer control and complications of salvage local therapy after failure of radiotherapy for prostate cancer: A systematic review. Semin Radiat Oncol. 2013;23:222-34.

29. Matei DV, et al. Salvage Radical Prostatectomy after External Beam Radiation Therapy: A Systematic Review of Current Approaches. Urol Int. 2015;94:373-82

30. Peters $\mathrm{M}$, et al. Patterns of outcome and toxicity after salvage prostatectomy, salvage cryosurgery and salvage brachytherapy for prostate cancer recurrences after radiation therapy: a multi-center experience and literature review. World J Urol. 2013;31:403-9.

31. Janoray G, Reynaud-bougnoux A, Ruffier-loubière A, Bernadou G. Stereotactic body re-irradiation therapy for locally recurrent prostate cancer after external-beam radiation therapy : Initial report Ré-irradiation stéréotaxique robotisée de récidive locale de cancer de prostate après radiothérapie externe : résultats p. Cancer/Radiother. 2016;20:275-81.

32. Duijzentkunst DAS, Peters M, Van Der Voort van Zyp JRN, Moerland MA van Vulpen M. Focal salvage therapy for local prostate cancer recurrences after primary radiotherapy : a comprehensive review. World J Urol. 2016. doi:10.1007/s00345-016-1811-9.

33. Kaljouw E, Pieters BR, Kovács G, Hoskin PJ. A Delphi consensus study on salvage brachytherapy for prostate cancer relapse after radiotherapy, a Uro-GEC study. Radiother Oncol. 2015. doi:10.1016/j.radonc.2015.10.021. 